\title{
Polynomial model of the inhibition mechanism of thiourea derivatives
}

\author{
István Lukovits, ${ }^{*}$ Imre Bakó, Abdul Shaban and Erika Kálmán \\ Central Research Institute for Chemistry, Hungarian Academy of Sciences, P.O. Box 17, \\ H-1525 Budapest, Hungary
}

(Received 7 November 1996; in revised form 15 February 1997)

\begin{abstract}
Interaction of corrosion inhibitors with metal surfaces in acidic solutions was modelled by using a polynomial model based on the Langmuir adsorption isotherm. The curve-fitting procedure yielded a regression equation with a multiple correlation coefficient equal to 0.982. (C) 1997 Elsevier Science Ltd
\end{abstract}

Key words: Simplex method, gradient method, quantum chemistry, molecular surface area.

\section{INTRODUCTION}

Corrosion inhibitors form a protective layer on the metal surface. The layer may be monomolecular or it may be fairly thick-referred to as a $21 / 2$ dimensional layer-consisting of the inhibitor and several compounds or ions. The inhibitors decrease the corrosion rate by various mechanisms: (1) the access of oxygen to the metal surface may be blocked; (2) the rate of dissolution of the protective layer on the metal surface may be reduced; (3) the dissolution of the metal may be blocked. The metal surface is polycrystalline and is covered by various oxidation products. Because of this complexity it is impossible to estimate the amount of adsorbed inhibitors by using parameters derived from monocrystalline experiments. The complexity of the inhibition process explains why statistical approaches were used in order to explain the variation of the efficiency of inhibition in terms of molecular parameters [1-7]. The derived equations were obtained by using the multiple linear regression analysis and are related to "fixed dose" corrosion inhibition. It has been shown that the statistics may significantly be improved if instead of "fixed dose" efficiencies, figures related to multiple doses were considered, but for the latter investigations more complicated non-linear regression equations have to be derived [8].

*Author to whom correspondence should be addressed.
In a recent study [9] inhibition efficiencies of thiourea derivatives were investigated in terms of molecular parameters, assuming that the amount of inhibitor molecules adsorbed on the metal surface can be estimated by using the Langmuir adsorption isotherm. The adsorption constant was determined a posteriori by using a non-linear regression (curvefitting) technique, and it is an average of the different constants related to various crystalline faces and oxidation products on the surface.

In this paper we propose a model that assumes that inhibition efficiency $E$ is a function of the "coverage" $A$ obtained by assuming a Langmuir isotherm. $E$ was expanded in terms of $A$. The results indicated that the systematic deviation from the ideal $\left(45^{\circ}\right)$ straight line [9] disappears if the Langmuir isotherm was replaced by our "polynomial model" and the error sum of squares was about $60 \%$ of that obtained with the model based on the Langmuir isotherm.

\section{METHOD}

The experimental data related to the inhibition efficiency of thiourea and several of its derivatives, were taken from the literature [10] and are listed in Table 1. The efficiencies were measured in acidic solution on mild steel (UNS G10080 carbon steel). There are 65 experimentally determined efficiencies altogether.

All molecules (Table 1) belong to the same "parent structure". The effect of the substituents 
Table 1.

Thiourea derivatives: Experimental efficiency of inhibition on mild steel in acidic solution [10]

\begin{tabular}{|c|c|c|c|}
\hline No. & $\begin{array}{l}\text { Molecule } \\
\text { (structure) }\end{array}$ & $\begin{array}{c}\text { Concentration } \\
(\mathrm{mol} / \mathrm{l})\end{array}$ & $\begin{array}{l}\text { Efficiency inhibition } \\
\text { (experimental) }\end{array}$ \\
\hline 1. & Thiourea & $3.2 \times 10^{-5}$ & 0.13 \\
\hline 2. & $\left(\mathrm{H}_{2} \mathrm{~N}-\mathrm{CS}-\mathrm{NH}_{2}\right.$ & $1.0 \times 10^{-4}$ & 0.23 \\
\hline 3. & & $2.5 \times 10^{-4}$ & 0.36 \\
\hline 4. & & $6.3 \times 10^{-4}$ & 0.68 \\
\hline 5. & & $1.0 \times 10^{-3}$ & 0.77 \\
\hline 6. & & $1.4 \times 10^{-3}$ & 0.82 \\
\hline 7. & & $5.0 \times 10^{-3}$ & 0.97 \\
\hline 8. & & $5.0 \times 10^{-2}$ & 0.96 \\
\hline 9. & & $1.0 \times 10^{-1}$ & 0.90 \\
\hline 10. & & $1.3 \times 10^{-1}$ & 0.88 \\
\hline 11. & Allylthiourea & $1.3 \times 10^{-6}$ & 0.04 \\
\hline 12. & $\left(\mathrm{H}_{2} \mathrm{~N}-\mathrm{CS}-\mathrm{NH}-\mathrm{CH}_{2} \mathrm{CH}=\mathrm{CH}_{2}\right)$ & $5.6 \times 10^{-6}$ & 0.09 \\
\hline 13. & & $1.0 \times 10^{-5}$ & 0.13 \\
\hline 14. & & $1.3 \times 10^{-5}$ & 0.18 \\
\hline 15. & & $2.2 \times 10^{-5}$ & 0.24 \\
\hline 16. & & $5.0 \times 10^{-5}$ & 0.38 \\
\hline 17. & & $1.0 \times 10^{-4}$ & 0.61 \\
\hline 18. & & $1.8 \times 10^{-4}$ & 0.94 \\
\hline 19. & & $7.9 \times 10^{-4}$ & 0.97 \\
\hline 20. & & $1.0 \times 10^{-2}$ & 0.98 \\
\hline 21. & & $1.0 \times 10^{-1}$ & 0.99 \\
\hline 22. & $\mathbf{N}, \mathbf{N}^{\prime}$-diethylthiourea & $1.8 \times 10^{-5}$ & 0.28 \\
\hline 23. & $\left(\mathrm{C}_{2} \mathrm{H}_{5} \mathrm{HN}-\mathrm{CS}-\mathrm{NHC}_{2} \mathrm{H}_{5}\right)$ & $7.9 \times 10^{-5}$ & 0.65 \\
\hline 24. & & $1.3 \times 10^{-4}$ & 0.80 \\
\hline 25. & & $1.8 \times 10^{-4}$ & 0.92 \\
\hline 26. & & $3.2 \times 10^{-4}$ & 0.95 \\
\hline 27. & & $3.5 \times 10^{-4}$ & 0.96 \\
\hline 28. & & $1.4 \times 10^{-3}$ & 1.0 \\
\hline 29. & & $3.5 \times 10^{-3}$ & 1.0 \\
\hline 30. & $\mathrm{~N}, \mathrm{~N}^{\prime}$ diisopropylthiourea & $1.6 \times 10^{-5}$ & 0.36 \\
\hline 31. & $\left(\mathrm{C}_{3} \mathrm{H}_{7} \mathrm{HN}-\mathrm{CS}-\mathrm{NHC}_{3} \mathrm{H}_{7}\right)$ & $3.5 \times 10^{-5}$ & 0.52 \\
\hline 32. & & $5.6 \times 10^{-5}$ & 0.60 \\
\hline 33. & & $7.1 \times 10^{-5}$ & 0.77 \\
\hline 34. & & $1.8 \times 10^{-4}$ & 0.95 \\
\hline 35. & & $1.3 \times 10^{-3}$ & 0.995 \\
\hline 36. & Penylthiourea & $1.0 \times 10^{-5}$ & 0.12 \\
\hline 37. & $\left(\mathrm{H}_{2} \mathrm{~N}-\mathrm{CS}-\mathrm{NH}-\mathrm{C}_{6} \mathrm{H}_{5}\right)$ & $1.0 \times 10^{-4}$ & 0.32 \\
\hline 38. & & $1.6 \times 10^{-4}$ & 0.55 \\
\hline 39. & & $2.5 \times 10^{-4}$ & 0.60 \\
\hline 40. & & $4.0 \times 10^{-4}$ & 0.74 \\
\hline 41. & & $6.3 \times 10^{-4}$ & 0.90 \\
\hline 42. & & $1.0 \times 10^{-3}$ & 0.97 \\
\hline 43. & & $2.2 \times 10^{-3}$ & 0.98 \\
\hline 44. & & $3.5 \times 10^{-3}$ & 0.98 \\
\hline 45. & & $4.0 \times 10^{-1}$ & 0.98 \\
\hline 46. & Thiocarbanilide & $3.2 \times 10^{-6}$ & 0.12 \\
\hline 47. & $\left(\mathrm{C}_{6} \mathrm{H}_{5} \mathrm{HN}-\mathrm{CS}-\mathrm{NHC}_{6} \mathrm{H}_{5}\right)$ & $1.3 \times 10^{-5}$ & 0.15 \\
\hline 48. & & $3.2 \times 10^{-5}$ & 0.29 \\
\hline 49. & & $5.0 \times 10^{-5}$ & 0.46 \\
\hline 50. & & $6.3 \times 10^{-5}$ & 0.52 \\
\hline 51. & & $1.0 \times 10^{-4}$ & 0.80 \\
\hline 52. & & $1.3 \times 10^{-4}$ & 0.895 \\
\hline 53. & & $2.2 \times 10^{-4}$ & 0.97 \\
\hline 54. & & $3.2 \times 10^{-4}$ & 0.99 \\
\hline 55. & & $6.3 \times 10^{-4}$ & 0.995 \\
\hline 56. & & $1.0 \times 10^{-3}$ & 1.0 \\
\hline 57. & Sym-di-o-tolylthiourea & $1.0 \times 10^{-5}$ & 0.21 \\
\hline 58. & $\left(\mathrm{CH}_{3} \mathrm{C}_{6} \mathrm{H}_{4} \mathrm{HN}-\mathrm{CS}-\mathrm{NHC}_{6} \mathrm{H}_{4} \mathrm{CH}_{3}\right)$ & $2.0 \times 10^{-5}$ & 0.32 \\
\hline 59. & & $3.2 \times 10^{-5}$ & 0.41 \\
\hline 60. & & $4.5 \times 10^{-5}$ & 0.64 \\
\hline 61. & & $5.6 \times 10^{-5}$ & 0.78 \\
\hline 62. & & $6.3 \times 10^{-5}$ & 0.94 \\
\hline 63. & & $2.0 \times 10^{-4}$ & 0.99 \\
\hline 64. & & $3.2 \times 10^{-4}$ & 0.995 \\
\hline 65. & & $4.5 \times 10^{-4}$ & 0.995 \\
\hline
\end{tabular}


Table 2.

Calculated quantum chemical indices [12] and areas of the molecular surfaces and values of $\log P$ [13] of thiourea derivatives considered in this work

\begin{tabular}{lccccr}
\hline Molecule & $\begin{array}{c}E_{\text {HOMO }} \\
(\mathrm{eV})\end{array}$ & $\begin{array}{c}E_{\text {LuMo }} \\
(\mathrm{eV})\end{array}$ & $\begin{array}{c}\mu \\
(\text { Debye })\end{array}$ & $\begin{array}{c}S \\
\left(\AA^{2}\right)\end{array}$ & $\log P$ \\
\hline Thiourea & -8.524 & 0.365 & 5.628 & 211 & -0.31 \\
Allylthiourea & -8.447 & 0.207 & 5.112 & 292 & 0.84 \\
N,N'-diethylthiourea & -8.277 & 0.250 & 4.747 & 334 & 1.19 \\
N,N'-diisopropylthiourea & -8.188 & 0.415 & 4.823 & 384 & 2.02 \\
Phenylthiourea & -8.439 & -0.176 & 4.803 & 326 & 2.29 \\
Thiocarbanilide & -8.476 & -0.485 & 5.090 & 444 & 3.33 \\
Sym-di-o-tolythiourea & -8.426 & -0.433 & 5.137 & 470 & 3.76 \\
\hline
\end{tabular}

was taken into account by using AM1 semiempirical quantum chemical calculations [11, 12] using full geometry optimization. The following quantum chemical indices were considered: the energy of the highest occupied molecular orbital ( $\left.E_{\text {HOMO }}\right)$, energy of the lowest unoccupied molecular orbital ( $\left.E_{\mathrm{LUMO}}\right), \Delta=E_{\mathrm{HOMO}}-E_{\mathrm{LUMO}}$ and the dipole moment $\mu$. The area of the molecular surface $S$ and the values of $\log P$ (where $P$ denotes the $n$-octanol/ water partition coefficient) were computed by using another standard computer program [13]. Table 2 lists the actual molecular parameters used in the present investigation.

The Langmuir adsorption is a starting point for our model. Coverage $\Lambda$ is a function of the adsorption constant $b$ and concentration $c$ of the adsorbed compound:

$$
\Lambda=b c /(1+b c) .
$$

Note that $A$ indicates the coverage due to the Langmuir adsorption isotherm, the actual coverage will be denoted by $\theta$. It was assumed that coverage is equal to the experimental efficiency of inhibition E:

$$
E \approx \theta \text {. }
$$

The present postulate has been used--sometimes tacitly - many times and it may also be found in a textbook [14], although coverage is not equivalent to inhibition efficiency.

The adsorption constant $b$ is a (linear) function of the molecular parameters:

$$
b=A_{1} X_{1}+A_{2} X_{2}+\cdots+A_{\mathrm{k}} X_{\mathrm{k}}+C
$$

where $X_{1}, X_{2}, \ldots, X_{\mathbf{k}}$ denote $k$ different molecular indices and $A_{1}, A_{2}, \ldots, A_{\mathrm{k}}$ are the regression coefficients and $C$ is the intercept. $b$ depends on the molecular structure and its properties alone, but it does not depend on the concentration. The right hand site of equation (3) may be used to replace $b$ in equation (1), and by using the assumption of equation (2) we obtain:

$$
E \approx A=\frac{\left(A_{1} X_{1}+A_{2} X_{2}+\cdots+A_{\mathrm{n}} X_{\mathrm{n}}+C\right) c}{1+\left(A_{1} X_{1}+A_{2} X_{2}+\cdots+A_{\mathrm{n}} X_{\mathrm{n}}+C\right) c}
$$

Regression coefficients $A_{1}, A_{2}, \ldots, A_{\mathrm{k}}$ and $C$ can be obtained from known efficiencies (coverages) and the related concentrations $c$. A Langmuir type function is valid only if adsorption takes place on a well defined smooth surface, a condition which is not fulfilled in partly corroded, polycrystalline, and rough metal surfaces. Therefore the calculated regression coefficients are associated with this rather "non-ideal" surface.

If experimental efficiencies and the corresponding concentrations are known, the regression parameters can (see below) be obtained, provided that the number of experiments is much higher than the number of unknown parameters $[8,9]$.

We have assumed that a fourth order polynomial in terms of $A$ is sufficient to approximate $E$. The function has the following features; 1. If $A=0$ then $E=0,2$. If $A=1$ then $E=1$. The following polynomial takes these restrictions into account:

$$
\begin{aligned}
E= & B_{1} A+B_{2} A^{2}+B_{3} A^{3} \\
& +\left(1-B_{1}-B_{2}-B_{3}\right) A^{4},
\end{aligned}
$$

where $B_{1}, B_{2}$ and $B_{3}$ are regression coefficients that have to be determined from experimental data. There are $k+4$ regression coefficients to be determined $\left(A_{1}, A_{2}, \ldots, A_{\mathrm{k}}, C, B_{1}, B_{2}\right.$ and $\left.B_{3}\right)$. The degrees of freedom (ie number of experimental data minus the number of parameters obtained through the fitting procedure) is equal to $N-k-1$ in the case where a Langmuir isotherm type function is fitted and it is equal to $N-k-4$, if a polynomial type function is fitted.

The curve fitting procedure was done in two steps. First the regression coefficients were obtained by using the Simplex technique [15], which is not very sensitive to the starting values of the parameters. This step was followed by a second curve fitting procedure using the gradient technique [16]. The results of the Simplex calculation were used as 
starting values of the gradient method. The reason for the second procedure was to obtain the errors of the regression coefficients, which could not be obtained by using the Simplex technique alone.

\section{RESULTS}

The best regression equation obtained by using the "Langmuir coverages" was:

$$
\begin{gathered}
E=\left(43678 E_{\text {LUMO }}+250 S-65671\right) c / \\
{\left[1+\left(43678 E_{\text {LUMO }}+250 S-65671\right) c\right]} \\
N=65, \quad R=0.971,
\end{gathered}
$$

where $R$ denotes the multiple correlation coefficient and $N$ is the total number of experimental efficiencies. The errors of the regression coefficients are as follows (the respective regression coefficients are listed once more in parentheses for the sake of clarity): \pm 6900 (43678), \pm 27 (250), \pm 8209 (-65671). All regression coefficients were significant at the $p \leqslant 0.01$ level, where $p$ denotes the level of significance [17]. The experimental and calculated efficiencies are shown in Fig. 1. Note the systematic deviation from the ideal $\left(45^{\circ}\right)$ straight line, which reflects the insufficiency of the Langmuir model. The results of other, less successful attempts are summarized in Table 3 . Only the independent variables and the multiple correlation coefficients were listed for the sake of brevity.

The polynomial model, based on $E_{\mathrm{LUMO}}$ and $S$, yielded the following non-linear regression equation:

$$
\begin{gathered}
A=\left(58136 E_{\text {LUMO }}+335 S-88168\right) \\
c /\left[1+\left(58136 E_{\text {LUMO }}+335 S-88168\right) c\right]
\end{gathered}
$$

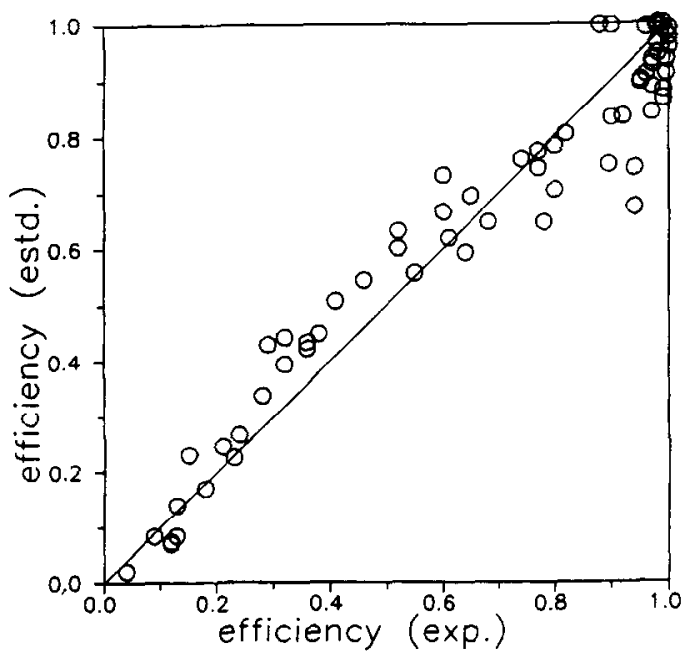

Fig. 1. Estimate (equation (6)) and experimental efficiencies of thiourea derivatives.
Table 3.

Multiple correlation coefficients obtained by using a Langmuir type function, equation (3), in terms of various sets of independent variables

\begin{tabular}{lc}
\hline $\begin{array}{l}\text { Combination of the } \\
\text { molecular indices }\end{array}$ & $R$ \\
\hline$E_{\text {HOMO }}$ & 0.922 \\
$E_{\text {LUMO }}$ & 0.889 \\
$\Delta$ & 0.884 \\
$\mu$ & 0.926 \\
$S$ & 0.949 \\
$\log P$ & 0.934 \\
$E_{\text {HOMO }} E_{\text {LUMO }}$ & 0.948 \\
$E_{\text {HOMO }} \mu$ & 0.930 \\
$E_{\text {HOMO }} S$ & 0.956 \\
$E_{\text {HOMO }} \log P$ & 0.950 \\
$E_{\mathrm{LUMO}}, \mu$ & 0.933 \\
$E_{\mathrm{LUMO}}, S$ & 0.971 \\
$E_{\mathrm{LUMO}}, \log P$ & 0.952 \\
$\Delta, \mu$ & 0.934 \\
$\Delta, S$ & 0.951 \\
$\Delta, \log P$ & 0.942 \\
$\mu, S$ & 0.949 \\
$\mu, \log P$ & 0.940 \\
$S, \log P$ & 0.969 \\
\hline
\end{tabular}

and

$$
\begin{gathered}
E=1.65 \Lambda-6.20 A^{2}+11.50 A^{3}-5.95 \Lambda^{4} \\
N=65, R=0.982,
\end{gathered}
$$

where the errors of the regression coefficients are as follows (the respective regression coeffcients are listed once more in parentheses): \pm 13378 (58136), \pm 73 (335), \pm 19419 (-88168), $\pm 0.36 \quad(1.65), \quad \pm 2.03 \quad(-6.20), \quad \pm 3.01 \quad$ (11.50). Note that the coefficient of the fourth order term $\left(A^{4}\right)$ was obtained by the following calculation:

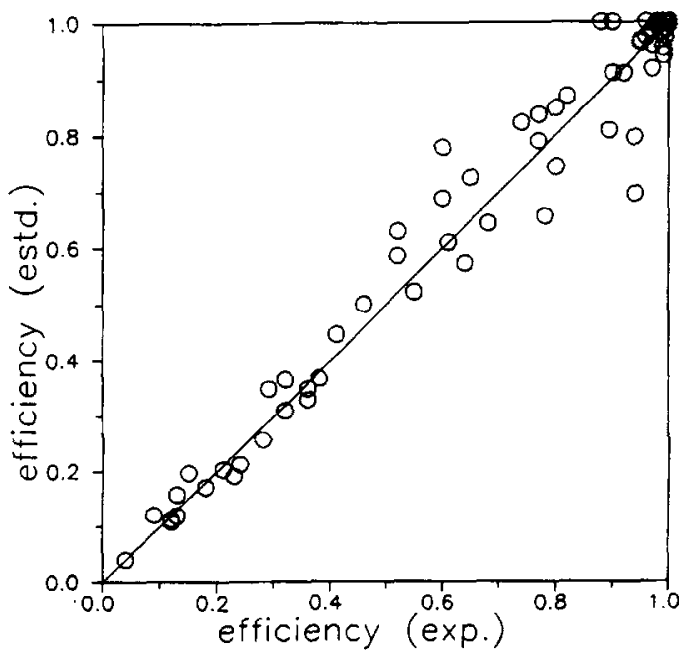

Fig. 2. Estimated (equations (7) and (8)) and experimental efficiencies of thiourea derivatives. 
$-5.95=1.0-1.65+6.20-11.50$. The experimental and calculated efficiencies are listed in Fig. 2.

A second combination of variables, namely $S$ and $\log P$ was found to yield nearly as good a Langmuir function as $E_{\mathrm{LUMO}}$ and $S$ in equation (6).

$$
\begin{gathered}
A=(347 S-13854 \log P-74486) c / \\
{[1+(347 S-13854 \log P-74486) c]} \\
N=65, R=0.969,
\end{gathered}
$$

and the errors of the regression coefficients are (listed in the same manner as in equation (6)): \pm 39 (347), $\pm 1954(-13854)$ and $\pm 8790(-74486)$. Again the polynomial approach yielded nearly as good results as found by using variables $E_{\mathrm{LUMO}}$ and $S$ in equation ( 7 and 8 ).

$$
\begin{aligned}
& A=(410 S-16282 \log P-87994) c / \\
& {[1+(410 S-16282 \log P-87994) c]}
\end{aligned}
$$

and

$$
\begin{gathered}
E=1.73 \Lambda-6.20 \Lambda^{2}+11.52 \Lambda^{3}-6.05 \Lambda^{4} \\
N=65, R=0.981,
\end{gathered}
$$

and the respective errors are: $\pm 83(410), \pm 3388$ (-16282), \pm 17838 (-87994), \pm 0.36 (1.73), \pm 2.03 $(-6.20)$ and \pm 3.08 (11.52). Although both $S$ and $\log$ $P$ were found to yield positive correlation coefficients when used as an independent variable in equation (4), the coefficient of $\log P$ is negative in equation (9), because of the interrelatedness between $S$ and $\log P$.

\section{DISCUSSION}

Both the Langmuir type function (equation (6)) and the polynomial type function (equation (7 and8)) seem to account for the variation in the experimental data in terms of molecular indices adequately, because the correlation coefficients and the regression coefficients were rather significant $(p<0.01)$. There is, however, a substantial difference in the error sum of squares (ie the deviation of the estimated values from the actual values), its value per degrees of freedom is equal to 0.0066 for equation (6) and 0.0041 for equations (7) and (8). The series expansion in terms of $A$ also removed the systematic alteration (Figs 1 and 2) of the estimated values obtained through equation (6). From these results we conclude that a Langmuir type function accounts only approximately for the adsorption of the inhibitors onto the partially corroded metal surfaces. Nevertheless it may be used in the polynomial model proposed in this paper.

From equation (7) we can see that increasing values of the energies of the lowest unoccupied orbitals favor inhibition, since the regression coeffcient is positive. This result means that charge transfer from the metal surface to the inhibitor molecules impairs efficiency. Increasing the area of the molecular surface enhances efficiency. This conclusion is not in accordance with the results of Arvia $e t$ al. [18], who found that on rough surfaces more voluminous ligands tend to cover a lower portion of the surface because of the "exclusion volume effects". This means that increasing the roughness of the adsorbent causes more voluminous molecules (and therefore molecules with a larger surface area) to have less access to the surface because the inolecules cannot enter the small cavities on this surface. Our results might indicate that in this series of thiourea derivatives the molecules are too small to produce the "exclusion volume effect".

The same conclusion may be drawn from equation (10). Increasing the molecular surface favors inhibition, whereas larger values of the partition coefficients might decrease efficiency.

The present model is clearly insufficient if there are retardation effects, ie at low concentrations efficiency does not increase with increasing concentration of the inhibitor. Retardation has not been observed with the experiments used in this study. If retardation appears, the model will have to be changed to take this phenomenon into account. The results may be used to predict the efficiency of new, and even as yet not synthesized, thiourea derivatives.

\section{ACKNOWLEDGEMENTS}

This work was supported by the National Research Fund (OTKA), No. T019785 and T017626.

\section{REFERENCES}

1. P. Dupin, D. A. Vilovia-Vera, A. de Savignac, A. Lattes, P. Haicour, in Proceedings 5th European Conference on Corrosion Inhibitors (Edited by $G$. Trabanelli), p. 301 . Università degli studi di Ferrara, Ferrara (1980).

2. F. B. Growcock, Corrosion 45, 1003 (1989)

3. F. B. Growcock, W. W. Frenier and P. A. Andreozzi, Corrosion 45, 1007 (1989).

4. P. G. Abdul-Ahad and S. H. F. Al Madfai, Corrosion 45,978 (1989).

5. J. I. Kuznetsov and L. A. Valuev, B. Electrochem. 4, 393 (1987).

6. J. I. Kuznetsov, S. Oleynik V. and S. S. Vesely, $B$. Electrochem. 3, 591 (1987).

7. J. I. Kuznetsov, B. Electrochem. 6, 571 (1990).

8. I. Lukovits, E. Kálmán and G. Pálinkás, Corrosion 51, 201 (1995).

9. I. Lukovits, K. Pálfi, I. Bakó, E. Kálmán, Corrosion, (in press).

10. I. Singh, Corrosion 49, 473 (1993).

11. M. J. S. Dewar, E. G. Zoebisch, E. H. Healy and J. P. Stewart, J. Am. Chem. Soc. 107, 3902 (1985).

12. Mopac, Release 6.0, J. J. P. Stewart, University of Austin, TX, 1983. 
13. ChemPlus(iil, Version 1.5. Hypercube Inc., Waterloo, Ontario, Canada, 1993-1995.

14. I. L. Rosenfeld, Corrosion Inhibitors, p. 127. McGrawHill, New York (1981).

15. J. A. Nelder and R. Mead, Computer J. 7, 308 (1965).

16. H. Rosenbrock, Computer J. 3, 175 (1960).
17. G. W. Snedecor and W. G. Cochran, Statistical Methods, p. 117. Iowa State University Press, Ames (1972).

18. M. Gómez M., M. P. Garcia, J. San Fabián, L. Vásquez, R. C. Salvarezza and A. J. Arvia, Langmuir 12,818 (1996). 